\title{
Forecasting seasonal rainfall characteristics and onset months over South Africa
}

\author{
Steven Phakula ${ }^{\mathrm{a}, \mathrm{b}}$ Willem A. Landman ${ }^{\mathrm{b}}$ and Asmerom F. Beraki ${ }^{\mathrm{c}}$ \\ ${ }^{a}$ South African Weather Service, Pretoria, South Africa, Email: \\ steven.phakula@weathersa.co.za, Tel: +27123676271 \\ ${ }^{\mathrm{b}}$ Department of Geography, Geoinfomatics and Meteorology, Pretoria, South Africa \\ ${ }^{\mathrm{c}}$ Council of Scientific and Industrial Research, Pretoria, South Africa
}

\begin{abstract}
Aspects of forecast skill in predicting seasonal characteristics using global climate models (GCMs) are assessed over South Africa. The GCMs output is configured to predict number of rainfall days at South African Weather Service stations exceeding predefined threshold values for the austral summer seasons and to predict the rainfall totals of the onset months of the rainy seasons for eight homogeneous rainfall regions of South Africa. Using canonical correlation analysis (CCA) as statistical downscaling technique through model output statistics, the forecast skill levels of coupled ocean-atmosphere and uncoupled atmospheric models are determined through retro-actively generated hindcasts. Both downscaled models have skill in predicting low and high number of rainfall days exceeding predefined thresholds for the austral summer seasons as well as rainfall totals of onset months. In addition to the forecast verification results, CCA pattern is performed to determine the dominating atmospheric circulation systems predicted to be controlling rainfall variations for the seasons and months of interest. CCA patterns for both the GCMs indicate that usually when there are anomalously negative (positive) predicted $850 \mathrm{hPa}$ geopotential heights over South Africa there are anomalously wet (dry) conditions over most parts of South Africa. The work has paved the way for the operational production of seasonal rainfall characteristics over South Africa in real time.
\end{abstract}


Keywords: Seasonal rainfall characteristics, Statistical downscaling, Retro-active hindcasts, Forecast verification.

\section{Introduction}

Southern Africa is characterized by significant rainfall variability on a range of temporal and spatial scales which have an impact on agriculture, water resources and the economy at large. The variability of rainfall makes the region vulnerable to extreme climate events (Shongwe et al. 2009). The most severe impacts of climate on human society and the natural environment over southern Africa are as a result of the extreme climate events such as droughts and floods, among others. The devastating floods in Mozambique during February/March 2000 and severe droughts of 1991/92, 2002/03 and 2003/04 in South Africa are the example of such extreme events (Cook et al., 2004). Therefore, better understanding of rainfall characteristics variables and atmospheric circulation systems that influence rainfall in the region is invaluable in order to provide detailed climate information to users. Different institutions in South Africa, including, the South African Weather Service (SAWS), are currently producing rainfall and temperature forecasts in real time at different time scales, including seasonal time scale. However, seasonal forecasts currently produced by SAWS do not always provide detailed information of specific needs to users. In addition, there has been an increasing demand for forecast of the weather statistics within a season (intraseasonal statistics) from agricultural and other user communities in decision making (Tadross et al., 2005; Hudson et al., 2011). The intra-seasonal forecasts are needed mainly because seasonal forecasts have limited benefits without medium-term information (Landman and Tennant, 2000). Moreover intra-seasonal forecasts may be able to bridge the gap between weather and seasonal forecasting, covering the time scale of about 10 to 60 days (Hudson et al., 2011). However, intra-seasonal forecasts are regarded as the most difficult to predict, but at the same time are of great importance for the economic and agricultural sectors at large. Although the chaotic nature of the atmosphere makes prediction of about two weeks to two months difficult (Luo and Wood, 2006), accurate prediction of weather statistics within a season may supplement existing seasonal forecast produced in real time. The objective of this study is to test the forecast skill of 
the coupled and uncoupled GCMs in predicting seasonal rainfall characteristics variables, viz. number of rainfall days exceeding pre-defined threshold values within the austral summer seasons as well as the onset months of the rainy seasons over South Africa.

\section{Data, models and methods}

a. Observed rainfall data

Quality controlled observed daily rainfall data from 563 rainfall stations distributed across South Africa obtained from SAWS are used. The climate databank of SAWS collates, maintains and runs a quality control process of South Africa's meteorological and climate data. In addition to quality control, only rainfall stations with no missing data from 1982 to 2009 are considered in this study. Monthly and 3-month seasonal rainfall totals as well as indices of the number of rainfall days exceeding $1,5,10,15,20,25,30$, 35, 40, 45 and 50mm for October-December (OND), November-January (NDJ), December-February (DJF) and January-March (JFM) seasons are calculated from the station rainfall data.

\section{b. $\quad$ Global model output data}

The $850 \mathrm{hPa}$ geopotential heights of the fourth generation of the ECHAM (ECHAM4.5, Roeckner et al., 1996; Beraki et al., 2016) atmospheric general circulation model (AGCM) developed at the Max Planck Institute for Meteorology (MPIM) in Hamburg, Germany, but administered by SAWS, is used in this study. The ECHAM evolved originally from the spectral weather prediction model of the European Centre for Medium Range Weather Forecasts (ECMWF; Simmons et al., 1989). The model is configured at a triangular spectral truncation 42 (T42) and at a resolution of about 2.8 degrees latitude and longitude with 19 vertical layers. The $850 \mathrm{hPa}$ geopotential heights of the coupled ocean-atmosphere general circulation model (OAGCM) developed in partnership between SAWS and the International Research Institute for Climate and Society (IRI), referred to as SAWS Coupled Model (SCM, Beraki et al. 2014) is also used. For this coupled configuration, the ECHAM4.5 AGCM feeds the ocean model called Modular Oceanic Model 
version 3 (MOM3; Pacanowski and Griffes, 1998) with heat, momentum, freshwater, and surface solar flux. In turn, MOM3 feeds the AGCM with SST information. Both the OAGCM and the AGCM are being used as operational seasonal forecast models at SAWS. The model's $850 \mathrm{hPa}$ geopotential heights are used because they have been found to be a good predictor for southern Africa summer rainfall seasons using model output statistics (MOS; Landman et al., 2014). The models data is from 1982 to 2009 and matching the observed data.

\section{c. $\quad$ Statistical downscaling: Model output statistics}

Because of the relatively low spatial resolution of the GCMs used in this study, downscaling of the global model output to capture local observations through the correction of systematic deficiencies in the global model is required (Landman and Beraki, 2012). Using the canonical correlation analysis (CCA) option of the Climate Predictability Tool (CPT; Mason and Tippet, 2016) the hindcast outputs of both the AGCM and the OAGCM are first statistically recalibrated (for the homogeneous regions) and downscaled (for the rainfall stations) to the observed seasonal rainfall totals and number of rainfall days exceeding $1 \mathrm{~mm}, 5 \mathrm{~mm}$, $10 \mathrm{~mm}, 15 \mathrm{~mm}, 20 \mathrm{~mm}, 30 \mathrm{~mm}, 40 \mathrm{~mm}$ and $50 \mathrm{~mm}$ within the seasons of interest as well as onset months of the rainy seasons over South Africa by using MOS. CCA is a multivariate statistical technique to determine optimal linear combination of two sets of data (predictors and predictands) that are highly correlated and is also used to build statistical downscaling models (Landman et al., 2009). MOS equations are developed to correct systematic biases in weather forecasts, as well as statistically correct climate model biases (Landman and Beraki, 2012; Wong et al., 2014). For climate models, MOS infers a correction function between model simulations and the corresponding observations and applies this correction function to a future simulation with the same model (Wong et al., 2014). In constructing the CCA model, the empirical orthogonal function (EOF) analysis is performed first because the predictor and the predictand fields contain a large number of highly correlated variables and few observations (Landman et al., 2001; 2009). EOF analysis provides a set of orthogonally-based vectors to convert a data set containing a large number of variables into a set containing fewer new variables. These new variables are linear combinations of the original ones, which 
are chosen to represent a larger fraction of the variability contained in the original data. The number of EOF modes to be retained in the analysis is determined using cross-validation forecast skill sensitivity tests (Landman and Tennant, 2000). The combination producing the highest area-average correlation is used as the best estimate of the number of predictor and predictand modes. The first CCA pair gives the maximum correlation between the two parameters, followed by the second CCA pair, and so on. CCA has the main advantage of selecting pairs of spatial patterns that are optimally correlated, making a physical interpretation of the connection between the observations and the retro-active forecasts or hindcasts possible (Busuioc et al., 2001).

\section{d. Downscaling procedures}

The CPT statistical downscaling tool have two options to train the MOS models, namely cross-validation and retro-active forecasts. Cross-validation has a tendency of overestimating forecast skill (Landman et al., 2001). On the other hand, retro-active forecast validation is a robust method to assess forecast model performance and give unbiased skill levels (Landman et al., 2001), and is therefore applied here. The retroactive procedure is as follows: firstly, usually half of the training sample is used as training period; secondly, reconstruct the model using that training period; thirdly, forecast the year that follows the last year of the training period; and lastly, repeat the process by adding one year to the training period and then predict subsequent year until a forecast has been made for each year of the training sample. For this study an initial training period of 14 years from 1982 to 1995 out of a training sample of 28 year from 1982 to 2009 is used to construct the model and to forecast the 1996 year. A training period of 1982 to 1996 is used to reconstruct a model and forecast the 1997 year. The process is repeated for each of the subsequent years until a forecast of each year has been made.

e. $\quad$ Verification of forecasts 
Forecast verification is the process of determining the quality of a forecast through assessment of the degree of similarity between that forecast and the observed conditions (Mandal et al., 2007). Verification of forecasts is mostly performed to check if there is a strong relationship between the forecasts and the observations and if the results provide an accurate indication of how good or bad subsequent forecast will be (Mason, 2008). For verifying seasonal probabilistic forecasts we focus here on the attributes of discrimination and reliability of the forecasts. For this purpose we use relative operating characteristics (ROC) and reliability diagrams, sometimes referred to as attribute diagrams (e.g. Landman et al., 2012; Landman and Beraki, 2012).

\section{i. Discrimination: ROC}

ROC is based on signal detection theory and seeks to measure the signal and noise contained in the forecast information in the form of hit or miss ratios when measured against performance level (Zhang and Casey, 2000). The idea of ROC comes from quality control and signal detection theory where the quality of performance is assessed by the correlation between hit and false alarm rates as the decision criterion varies (Zhang and Casey, 2000). ROC can be quantified by calculating the area beneath the ROC curve (Mason and Graham, 1999). The larger the area, the better the model skill. If the area is equal to or less than 0.5 of the whole (unit area), then the model is less skillful than a random or constant forecast. In this study the area underneath the ROC is used as a measure of discrimination.

\section{ii. Reliability: attributes diagrams}

Reliability or attributes diagram explain the resolution and reliability attributes which together determine the usefulness of probabilistic forecast systems (e.g. Brocker and Smith, 2007). Resolution measures the ability of a forecast system to resolve situations in which the observed frequency of the event is different to the climatological frequency, while reliability is a measure of the bias in predicted probabilities for the event, relative to the verified event frequency. A forecast with good reliability is closer to the perfect 
reliability line (diagonal line on the attributes diagram), while a forecast with good resolution has a wide range of frequency of observations corresponding to forecast probabilities. Resolution is considered the more fundamental of the two attributes, because reliability may generally be improved by calibration of the forecast probabilities, while resolution cannot. A forecast system that underestimate (overestimate) forecasts will have the forecast line positioned above (below) the perfect reliability line. The line halfway between climatology and perfect reliability is the no skill line, where the reliability and resolution are equal and the Brier skill score approaches zero (Wilks 2011). The histogram of forecasts in each probability bin shows the sharpness of the forecast.

\section{Results}

\subsection{Seasonal rainfall predictions}

The study first performs a skill assessment of the 3-months season in order to establish: 1) a baseline skill level which the skill levels of seasonal rainfall characteristics forecasts can be compared to, and 2) to see if the GCMs can replicate skill levels already established for seasonal forecasts over southern Africa (Landman et al., 2012). In this section the skill levels of both the OAGCM and the AGCM in predicting the 3-month seasonal rainfall totals for OND, NDJ, DJF and JFM over South Africa is evaluated.

\section{a. Relative operating characteristics}

ROC scores (Figure 1) show that both the models have skill (ROC scores $>0.5$ ) in predicting seasonal rainfall totals at different lead-times over South Africa, the skill is highest during NDJ and DJF seasons. For the OAGCM system, when predicting above-normal rainfall totals (wet conditions) highest scores are found at 3- and 4-month lead-times during NDJ seasons as well as at 2-month lead-time during DJF seasons. Above-normal or wet conditions is defined as when seasonal rainfall totals in an area is above the $67 \%$ tercile from the climatology. In predicting below-normal rainfall totals (dry conditions) highest scores are found at 3-month and 0-month lead-times during NDJ and DJF, respectively. Below-normal or dry 
conditions is defined as when seasonal rainfall totals in an area is below the $33 \%$ tercile from the climatology. The lowest scores of less than 0.5 are found at 1 - and 3-month lead-times in predicting wet conditions for OND seasons as well as at 3-month lead-time for JFM seasons. It is also shown that when predicting dry conditions the lowest scores are found at 3- and 4-month lead-times during OND seasons. For the AGCM prediction system, highest ROC scores in predicting wet conditions are found at 3-month lead-time during the NDJ seasons and at 0-, 2-, 3- and 4-month lead-times during DJF seasons as well as at 1-month lead-time during JFM seasons. In predicting dry conditions the highest scores are found at 2- and 3-month lead-times during NDJ as well as 4- and 1-month lead-times during DJF and JFM, respectively.

\section{b. Reliability diagrams}

Because the highest ROC scores (Figure 1) are found during NDJ and DJF only the reliability for these two seasons are shown. Reliability diagrams indicate that both the models are over-confident (under-confident) when predicting dry (wet) conditions during NDJ seasons (Figure 2), a result often found for South African seasonal rainfall forecasts (Landman et al., 2012, 2014). For DJF seasons, the reliability diagrams (Figure 3) indicate that both models are reliable in predicting wet conditions, while the forecasts for both systems are always over-confident when predicting dry conditions. In fact, it is a common problem for climate models to be over-confident in predicting rainfall over South Africa (e.g. Landman et al., 2012, 2014). The frequency histograms indicates that the forecasts for both systems lack sharpness when predicting both wet and dry conditions. In general, the forecasts display a lack of sharpness because forecasts rarely deviate much from the climatological value of 33.3\%. Moreover, low sharpness is common for South African rainfall prediction studies (Landman et al., 2012, 2014). The results presented in this section are in agreement with previous studies of seasonal rainfall predictions in South Africa (e.g. Landman et al., 2012)

and they subsequently show that the model configurations have been set up properly and from these configurations the predictability of additional statistics of the season can be tested.

c. Canonical correlation analysis patterns 
Again only the CCA pattern analysis for NDJ and DJF are performed and analyzed, for the same reason as in the previous section. CCA pattern results for both the OAGCM and the AGCM suggest that same physical mechanism of atmospheric circulation patterns are responsible for rainfall during all summer seasons considered. Because similar result for both models are found for the two seasons only the AGCM's CCA maps for DJF is shown here. It is found that when there are anomalously negative (positive) predicted 850hPa geopotential heights over South Africa, there are anomalously wet (dry) conditions for most parts of South Africa. This conclusion is obtained in the following way. CCA maps (Figure 4) show that during 1982/1983 and 2006/2007, for example, the predictor's spatial loadings are anomalously negative and the corresponding temporal scores are also negative. The product of the predictor's spatial loadings and the time scores is positive. During the same years over most parts of South Africa, the rainfall spatial loadings are positive and the temporal scores are negative, and their product is negative. This result implies that when there are anomalously positive $850 \mathrm{hPa}$ geopotential heights over South Africa there are anomalously dry conditions over South Africa. In fact, when there is a high-pressure system over the interior of South Africa it draws moisture out of the country and usually the country remains dry (Cook et al., 2004). Now during 1999/2000 and 2007/2008 there are anomalously negative predictor's spatial loadings over South Africa and the time scores are positive, and their product is negative. The rainfall spatial loadings over most parts of South Africa are anomalously positive and the time scores are positive. The product of rainfall spatial loadings and the time scores is positive. Likewise this implies that when there are anomalously negative $850 \mathrm{hPa}$ geopotential heights over South Africa there are anomalously wet conditions over most parts of South Africa. In fact, when there is a low-pressure system over the interior of South Africa during summer seasons, it advects moisture into the country and usually results in rainfall events (Cook et al., 2004). It can be established from the CCA pattern results that most of the years that are anomalously dry are associated with El Niño events (El Niño years are marked X in the temporal scores in Figure 4), whereas year that are anomalously wet are linked with La Niña events (La Niña years are marked O in the temporal scores in Figure 4). It is well documented that El Niño (La Niña) episodes are usually associated with anomalously dry (wet) conditions over the summer rainfall area of South Africa (e.g. Washington and 
Preston, 2006). Most notably, during the El Niño events of 1982/1983 and 1991/1992 South Africa experienced severe droughts and the 1999/2000 La Niña event left most of the summer rainfall areas of South Africa wetter than normal, including floods in some areas.

\subsection{Seasonal rainfall characteristics predictions}

\subsubsection{Rainfall days exceeding pre-defined thresholds}

In this section the skill levels of both the OAGCM and the AGCM at 1-month lead-time in predicting the high- and low-number of rainfall days exceeding 1, 5, 10, 15, 20, 30, 40 and 50mm are evaluated over South Africa. High number is defined as when number of rainfall days within a season in an area is above the $67 \%$ tercile from the climatology, whereas low number is defined as when number of rainfall days within a season in an area is below the $33 \%$ tercile from the climatology.

\section{a. Relative operating characteristics}

ROC scores (Figure 5) show that both models have the attribute of discrimination in predicting the number of days exceeding threshold values less than or equal to $20 \mathrm{~mm}$. The skill level is high during NDJ and DJF seasons for the OAGCM prediction system as compared to OND and JFM seasons, with highest ROC scores found in predicting high number of rainfall days exceeding 1 and $5 \mathrm{~mm}$ during DJF as well as $10 \mathrm{~mm}$ during NDJ. For the AGCM prediction system, the highest scores is found when predicting the high number of rainfall days exceeding 1 and 5mm during NDJ and DJF, respectively, as well as 10mm during JFM seasons. However, it is worth noting that in predicting the number of days exceeding $5 \mathrm{~mm}$ the ROC score is less than 0.5 for NDJ seasons. Furthermore, the skill level is decreasing with higher threshold values when predicting the high number of rainfall days. On the other hand, the skill level in predicting the low number of rainfall days is increasing with higher threshold values. The increasing of skill is due to the fact that there are fewer low number of rainfall days to count for higher thresholds. 


\section{b. Reliability diagrams}

The highest ROC scores (Figure 5) are found during DJF seasons for the OAGCM and during JFM seasons for the AGCM, hence only the reliability for them are shown. Reliability diagrams also indicate that both systems can reliably predict number of rainfall exceeding thresholds less or equal to $20 \mathrm{~mm}$, especially during DJF and JFM seasons. Reliability diagrams indicate that the OAGCM (Figure 6) and the AGCM (Figure not shown) are reliable in predicting high-number of rainfall days exceeding less than or equal to $20 \mathrm{~mm}$ for DJF seasons. It is also evident that the AGCM (Figure 7) and the OAGCM (Figure not shown) are reliable when predicting high number of rainfall days exceeding less or equal to $20 \mathrm{~mm}$ during JFM seasons. However, forecasts of the two forecasting systems tend to be over-confident when predicting low number of rainfall days exceeding pre-defined thresholds, particularly for higher threshold values, during DJF and JFM seasons.

\section{c. CCA patterns}

CCA patterns for both the OAGCM and the AGCM show that when there are anomalously negative (positive) predicted $850 \mathrm{hPa}$ geopotential heights over South Africa, there are anomalously high (low) number of rainfall days exceeding the pre-defined threshold values for DJF and JFM seasons over most parts of South Africa. Such a configuration is also found for modelling seasonal rainfall totals (cf. Figure 4). Only CCA pattern for the AGCM and thresholds exceeding 10mm during DJF seasons is shown here (Figure 8) because the results are similar for all the thresholds considered for both the OAGCM and the AGCM. Figure 8 show that when there are anomalously negative (positive) predicted $850 \mathrm{hPa}$ geopotential heights over South Africa, there are anomalously high (low) number of rainfall days exceeding 10mm for DJF seasons over most parts of South Africa. Refer to section 3.1 (c) for analysis on CCA patterns. It can be established that most of the years that are associated with low number of rainfall days are actually El Niño years (marked X in temporal scores in Figure 8) and usually during El Niño episodes large part of South Africa receives below-normal rainfall, sometimes even drought. This implies that for summer rainfall 
seasons during these El Niño events there are high frequency of high-pressure systems over the country suppressing rainfall activities and reducing the number of rainfall days. In contrast, during the La Niña years (marked $\mathrm{O}$ in temporal scores in Figure 8) large part of South Africa receive above-normal rainfall as well as floods at times. It can be established that during La Niña events there are high frequency of lowpressure systems over South Africa that are associated with ridging highs bringing moisture into the country resulting in wet conditions, in turn leading to high number of rainfall days.

\subsection{Onset months of the rainy season predictions}

This section evaluates the OAGCM and the AGCM forecasting systems in predicting rainfall totals of the onset months of the rainy seasons for the eight homogeneous rainfall regions of South Africa (Landman et al., 2009). The onset is defined here as the first month of a 3-month season on condition that the season consists of the wettest consecutive 3 months of the year as calculated over several decades (climatological values). According to this definition of onset, the onset month for Region 1 is May, October for Region 2 and 3, November for Region 4, 5 and 6, December and January for Region 7 and 8, respectively and are indicated with black arrows on the climatological annual rainfall circle (Figure 9). The models' forecast skill levels is again evaluated using ROC scores and reliability diagrams. CCA patterns are also performed in order to determine the dominating atmospheric circulation systems predicted to be controlling rainfall and subsequently the onset months of the main rainfall seasons.

\section{a. Relative operating characteristics}

The two forecasting systems have skill in predicting rainfall totals for the onset months of the rainy seasons of the eight homogeneous rainfall regions. The ROC scores bar graphs (Figure 10) indicate that the OAGCM is more skillful in predicting dry conditions as compared to wet conditions for the onset months of Region 1, 3, 4, 5 and 8, with highest ROC scores found for Region 1, 4 and 8. On the other hand, highest scores are found when predicting wet conditions for the onset months of Region 8 and 7, followed by 
Region 1 and 5. In fact, the OAGCM system in more skillful when predicting dry conditions as compared to wet conditions. For the uncoupled model, the highest scores are found when predicting wet conditions for the onset months for Region 5 followed by Region 4 and 1, whereas in predicting dry conditions highest scores are found in Region 2, 3, 5 and 8. In contrast to the OAGCM, the AGCM is more skillful when predicting wet conditions as compared to dry conditions for the onset months.

\section{b. Reliability diagrams}

Reliability diagrams indicate that both systems have some level of reliability in predicting wet and dry conditions for the onset months for the eight homogeneous rainfall regions. In fact, both models seem to perform better when predicting wet conditions as compared to dry conditions. However, the two forecasting systems again lack sharpness when predicting both wet and dry conditions for the onset months for the rainfall regions of South Africa. Only reliability diagrams for Region 1 (Figure 11) and Region 5 (Figure 12) arbitrarily selected are shown here. It must be noted however that Region 1 is a winter rainfall region and Region 5 is a summer rainfall region. Figure 11 show that both the models are reliable in predicting wet conditions and over-confident in predicting dry conditions for Region 1. Reliability diagram (Figure 12) indicate that the OAGCM (AGCM) forecasting system is under-confident (over-confident) in predicting wet conditions for the onset months for Region 5. On the other hand, both models are over-confident in predicting dry conditions.

\section{c. CCA pattern analysis}

CCA patterns indicate that there are different atmospheric circulation systems produced by the global models responsible for rainfall for different rainfall regions. Only CCA maps for Region 1 (Figure 13) and Region 5 (Figure 14) are again shown here. These two figures, although selected arbitrarily, do show the different dominating circulation systems responsible for skill for the two regions. CCA maps for Region 1 indicate that when there are anomalously negative (positive) predictor's spatial loadings for the OAGCM 
positioned southeast of South Africa, there are anomalously dry (wet) conditions for the onset months. During 1983, 1998 and 2006 for example, the predictor's spatial loadings are anomalously negative and their temporal scores are also negative. The product of the spatial loadings and their temporal scores is positive. The predictand loadings for the onset months are anomalously negative and their temporal scores are negative, and their product is positive. During 1982 and 2003 the predictor's loadings are anomalously negative and their temporal scores are positive, and their product is negative. The predictand loadings are anomalously negative and their temporal scores are positive, and their product is negative. This result implies that when there is a strong low- (high-) pressure system at $850 \mathrm{hPa}$ situated southeast of South Africa the onset months for Region 1 tend to be drier (wetter) than normal. On the other hand, the CCA pattern analysis for Region 5 indicate that when there are anomalously negative (positive) predictor's spatial loadings over South Africa extending to southwest Indian ocean (SWIO), there are anomalously wet (dry) conditions for the onset months. During 1982 and 2002 the predictor' spatial loadings are anomalously negative and their temporal scores are negative, and their product is positive. The predictand loadings are positive and their temporal scores are negative, and their product is negative. During 1986 and 1999 the predictor's loadings are anomalously negative and their temporal scores are positive, and their product is negative. The predictand loadings are positive and their temporal scores are positive, and their product is positive. This result mean that when there is a strong high (low) pressure system at $850 \mathrm{hPa}$ over South Africa extending to SWIO the onset months for Region 5 tend to be dry (wet).

\section{Discussion and Conclusions}

Forecast skill of the OAGCM and the AGCM are evaluated in predicting number of rainfall days exceeding pre-defined thresholds as well as rainfall totals of the onset months of the rainy seasons over South Africa. Although the aim of this research is to evaluate the models' ability in predicting seasonal rainfall characteristics and onset months of rainy seasons over South Africa, the models are first evaluated in predicting seasonal rainfall totals to determine whether the results of this study are comparable with previous studies of seasonal climate predictions. By evaluating seasonal predictability first it is 
subsequently shown that the model configurations have been set up properly and from these configurations additional properties of the season are predicted.

It was established that both the GCMs have skill in predicting the 3-month seasonal rainfall totals for OND, NDJ, DJF and JFM over South Africa. In fact the highest ROC scores are found during NDJ and DJF seasons. Reliability diagrams for NDJ seasons showed that both forecasting systems are over-confident (under-confident) in predicting dry (wet) conditions over South Africa. For DJF seasons the reliability diagrams indicated that both models have some level of reliability in predicting wet conditions, however the forecast systems are always over-confident in predicting dry conditions. CCA pattern analysis indicate that similar atmospheric circulation patterns produced by the global models are responsible for rainfall during summer seasons. It was established from CCA pattern analysis for both prediction systems that when there are strong high- (low-) pressure systems at the surface over South Africa, there are anomalously dry (wet) conditions for NDJ, DJF and JFM seasons over of South Africa.

It was also found that when predicting the number of rainfall days exceeding pre-defined threshold values over South Africa both forecasting systems also have skill. The ROC scores have shown that both models have the attribute of discrimination in predicting the number of days exceeding threshold values less than or equal to $20 \mathrm{~mm}$. The highest ROC scores are found during NDJ and DJF for the OAGCM system and during DJF and JFM for the AGCM system. The reliability diagrams also indicate that both systems can reliably predict number of rainfall exceeding thresholds less or equal to $20 \mathrm{~mm}$, especially during DJF and JFM seasons. CCA pattern analysis showed that when there are anomalously negative (positive) predicted 850hPa geopotential heights over South Africa, there are anomalously high (low) number of rainfall days exceeding the pre-defined threshold values for NDJ, DJF and JFM seasons over South Africa. Such configuration is also found for modelling seasonal rainfall totals.

The forecasting systems also have skill (ROC scores $>0.5$ ) in predicting rainfall totals for the onset months of the eight homogeneous rainfall regions of South Africa. The reliability diagrams also showed that both 
systems have some level of reliability in predicting rainfall totals for the onset months, but both the prediction systems lack sharpness. CCA maps showed that in general a variety of atmospheric circulation systems are responsible for rainfall for the onset months of the eight rainfall regions of South Africa. In fact, circulation systems produced by the OAGCM are different from the ones produced by the AGCM.

The format of seasonal forecasts issued by institutions such as SAWS has been for the most part focused on the likelihood of receiving predefined seasonal rainfall totals. That is, the forecasts have focused almost exclusively on predicting droughts or flood seasons as defined by accumulated rainfall totals over a season. Users of forecasts are interested to also find out what forecast for rainfall statistics within a season might be like. For this reason, this study was conducted to test if our current forecast models can in fact skillfully predict such statistics. Here the focus was on predicting the number of rainfall days exceeding predefined thresholds as well as the rainfall totals for the onset months of rainy seasons. The levels of skill in predicting these statistics are similar to what has been known, and again demonstrated here, for South Africa. This work has paved the way for SAWS to issue forecasts for such statistics, in addition to the traditional seasonal forecasts.

\section{Acknowledgements}

This work was funded by the Applied Centre for Climate and Earth Systems Science (ACCESS) and was also partially funded by South African Weather Service.

\section{References}

Beraki AF, DeWitt DG, Landman WA, Olivier C. 2014. Dynamical climate prediction using an oceanatmosphere coupled model developed in partnership between South Africa and the IRI. Journal of Climate 27: 1719-1741. 
Beraki AF, Landman WA, DeWitt D, Olivier C. 2016. Global dynamical forecasting system conditioned to robust initial and boundary forcings: Seasonal Context. International Journal of Climatology, DOI: 10.1002/joc.4643.

Brocker J, Smith LA. 2007. Increasing the reliability of reliability diagrams. Weather and Forecasting 22: 651-661.

Busuioc A, Chen D, Hellstrom C. 2001. Performance of statistical downscaling models in GCM validation and regional climate change estimates: Application for Swedish precipitation. International Journal of Climatology 21: 557-578.

Cook C, Reason CJC, Hewitson BC. 2004. Wet and dry spells within particularly wet and dry summers in the South African summer rainfall region. Climate Research 26: 17-31.

Hudson D, Alves O, Hendon HH, Marshall AG. 2011. Bridging the gap between weather and seasonal forecasting: intraseasonal forecasting for Australia. Quarterly Journal of the Meteorological Society 137: 673-689.

Landman WA, Tennant WJ. 2000. Statistical downscaling of monthly forecasts. International journal of Climatology 20: 1521-1532.

Landman WA, Mason SJ, Tyson PD, Tennant WJ. 2001. Retro-active skill of multi-tiered forecasts of summer rainfall over southern Africa. International Journal of Climatology 21: 1-19.

Landman WA, Kgatuke M-J, Mbedzi M, Beraki A, Bartman A, du Piesanie A. 2009. Performance comparison of some dynamical and empirical downscaling methods for South Africa from a seasonal perspective. International Journal of Climatology 29: 1535-1549.

Landman WA, Beraki A. 2012. Multi-Model forecast skill for mid-summer rainfall over southern Africa. International journal of Climatology 32: 303-314.

Landman WA, DeWitts D, Lee DE, Beraki A, Lotter D. 2012. Seasonal rainfall prediction skill over South Africa: One- versus two-tiered forecasting systems. Weather and Forecasting 27: 489-501. 
Landman WA, Beraki A, DeWitt D, Lötter D. 2014. SST prediction methodologies and verification considerations for dynamical mid-summer rainfall forecasts for South Africa, Water SA, 40(4), 615-622, http://dx.doi.org/10.4314/wsa.v40i4.6.

Luo L, Wood EF. 2006. Assessing the idealized predictability of precipitation and temperature in the NCEP Climate forecast System. Geophysical Research Letters 33: L04708, doi: 10.1029/2005GL025292, 2006. Mandal V, Utpal KD, Biplab KB. 2007. Precipitation forecast verification of the Indian summer monsoon with intercomparison of three diverse regions. Weather and Forecasting 22: 428-443.

Mason SJ. 2008. Understanding forecast verification statistics. Meteorological Applications 15: 31-40.

Mason SJ, Tippett MK. 2016. Climate Predictability Tool version 15.3, Columbia University Academic Commons. http://dx.doi.org/10.7916/D8NS0TQ6.

Mason SJ, Graham NE. 1999. Conditional probabilities, relative operating characteristics, and relative operating levels. Weather and Forecasting 14: 713-725.

Pacanowski RC, Griffes SM. 1998. MOM 3.0 manual. NOAA/Geophysical Fluid Dynamics Laboratory, Princeton, NJ, 608 pp.

Roeckner E, Coauthors. 1996. Simulation of present-day climate with the ECHAM4 model: Impact of model physics and resolution, Report No. 93, Max-Planck-Institut für Meteorologie, Hamburg, Germany, $171 \mathrm{pp}$.

Shongwe ME, Van Oldenborgh GJ, Van Den Hurk BJJM, De Boer B, Coelho CAS, Van Aalst MK. 2009. Projected changes in mean and extreme precipitation in Africa under global warming: Part I: Southern Africa. Journal of Climate 22: 3819-3837.

Simmons AJ, Burridge DM, Jarraud M, Girard C, Wergen W. 1989. The ECHAM medium-range prediction models: development of the numerical formulations and the impact of increased resolution. Meteorological Atmospheric Physics 40: 28-60.

Tadross MA, Hewitson BC, Usman MT. 2005. The international variability of the onset of the maize growing season over South Africa and Zimbabwe. Journal of Climate 18: 3356-3372. 
Washington R, and A. Preston, 2006: Extreme wet years over southern Africa: Role of the Indian Ocean sea surface temperatures. J. Geophys. Res. 111,D15104, doi:10.1029/2005JD006724.

Wilks DS. 2011. Statistical methods in the atmospheric sciences. $3^{\text {rd }}$ edition. Academic Press: Amsterdam. 676pp.

Wong G, Maraun D, Vrac M, Widmann M, Eden JM. 2014. Stochastic Model Output Statistics for bias correcting and downscaling precipitation including extremes. Journal of Climate 27: 6940-6959.

Zhang H, Casey T. 2000. Verification of probability forecasts. Weather and Forecasting 15: 0-89.

\section{Figure captions}
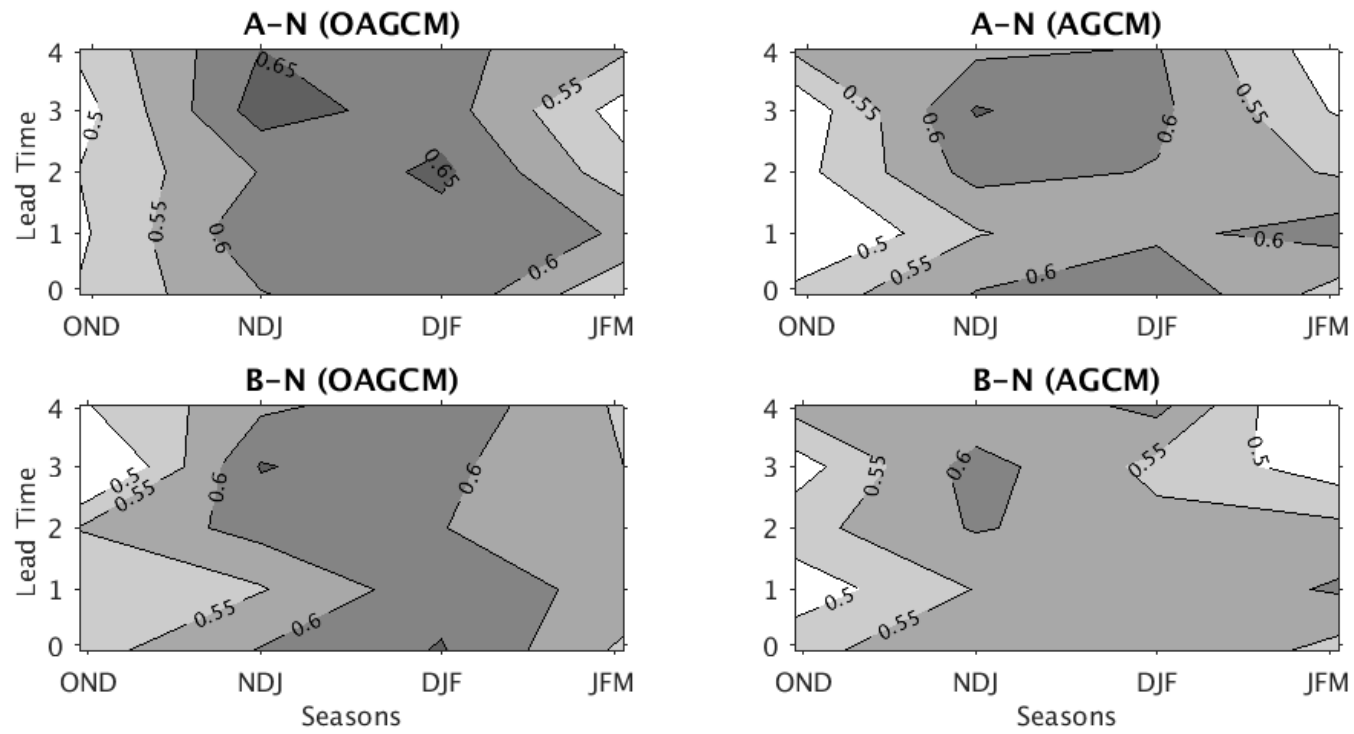

Figure 1. ROC scores of both the OAGCM (left panel) and the AGCM (right panel) at 0- to 4-month lead-times in predicting the seasonal rainfall for the summer rainy seasons overs the 14 years retro-active forecasts from 1996-2009. 

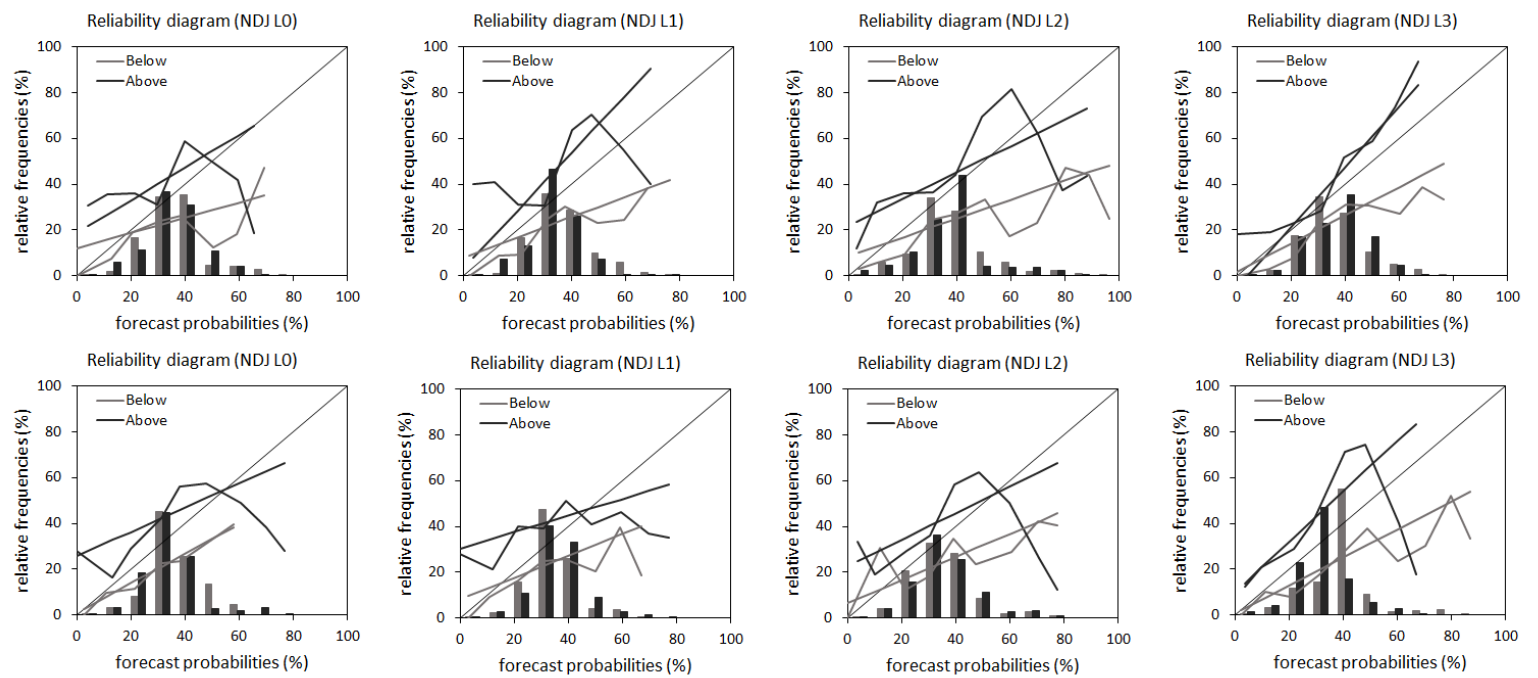

Figure 2. Reliability diagrams and frequency histograms for the OAGCM (top panel) and the AGCM (bottom panel) at 0- to 3-months lead-time (L0, L1, L2 and L3) in predicting above-normal and below-normal rainfall totals during NDJ seasons over 14 years retro-active forecasts from 1996 to 2009.
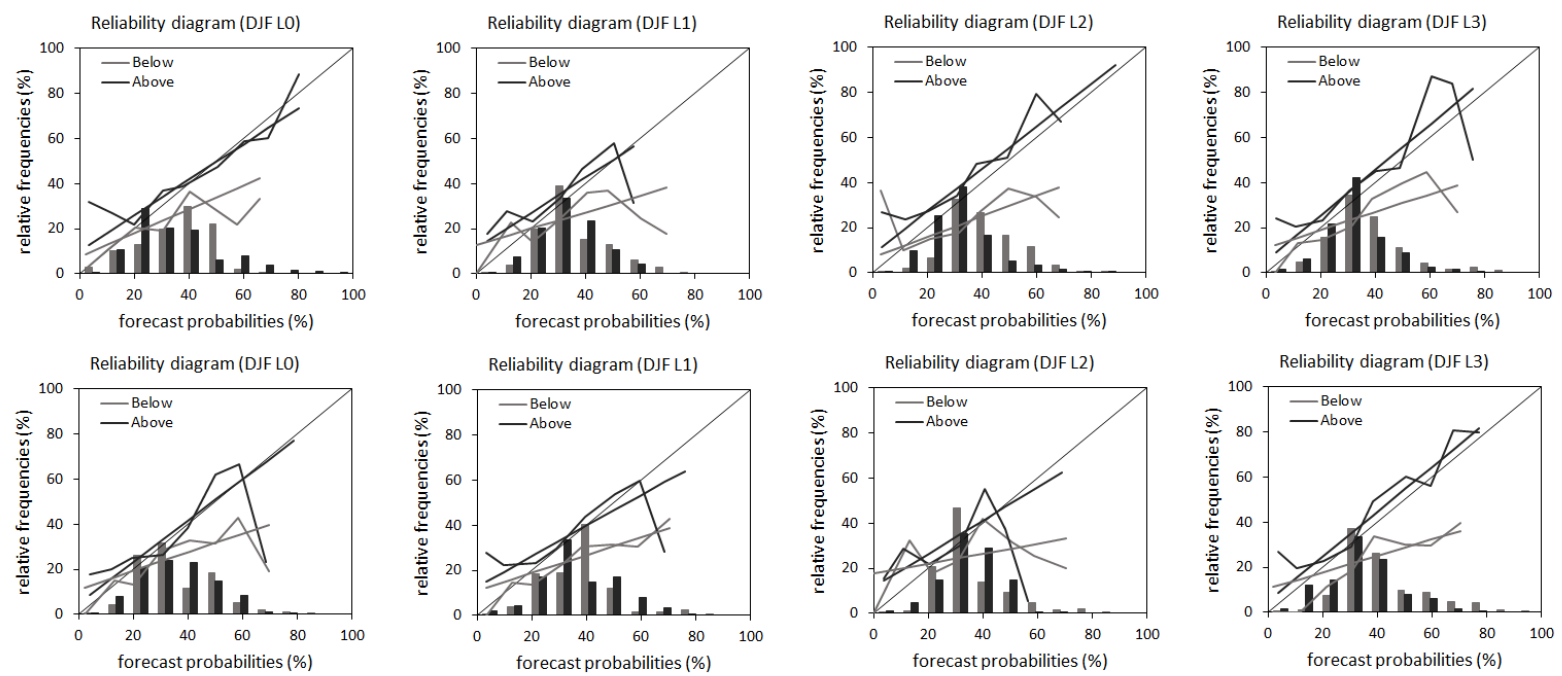

Figure 3. Reliability diagrams and frequency histograms for the OAGCM (top panel) and the AGCM (bottom panel) at 0- to 3-months lead-time (L0, L1, L2 and L3) in predicting above-normal and below-normal rainfall totals during DJF seasons over 14 years retro-active forecasts from 1996 to 2009. 

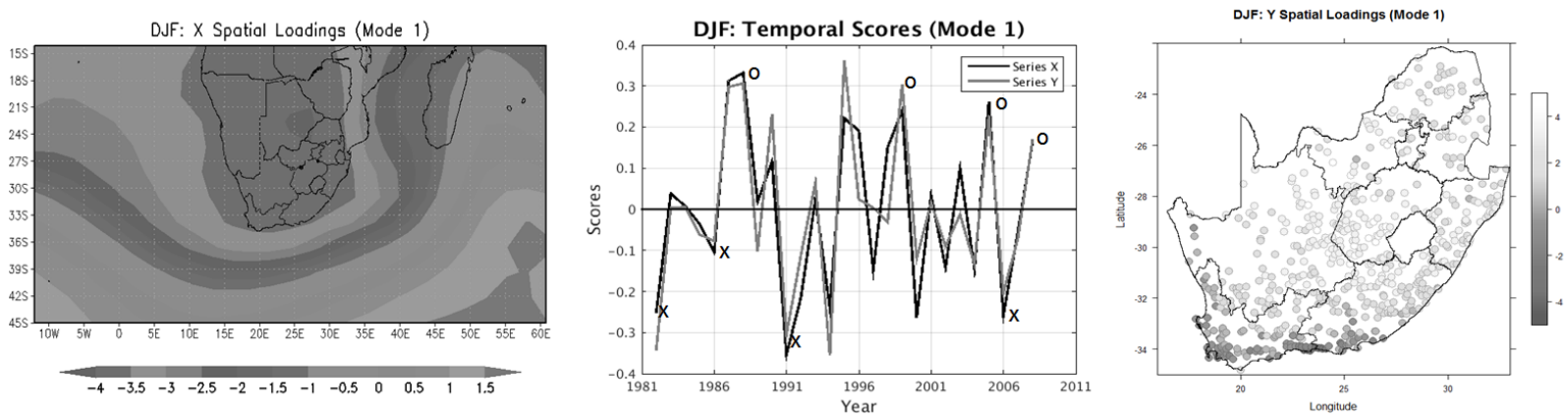

Figure 4. Mode $1 \mathrm{CCA}$ maps of the $850 \mathrm{hPa}$ geopotential heights of the AGCM at 1-month lead-time and the downscaled seasonal rainfall totals for DJF seasons. The X spatial loadings and the Y spatial loadings maps are for the predictors and the predictands, respectively.
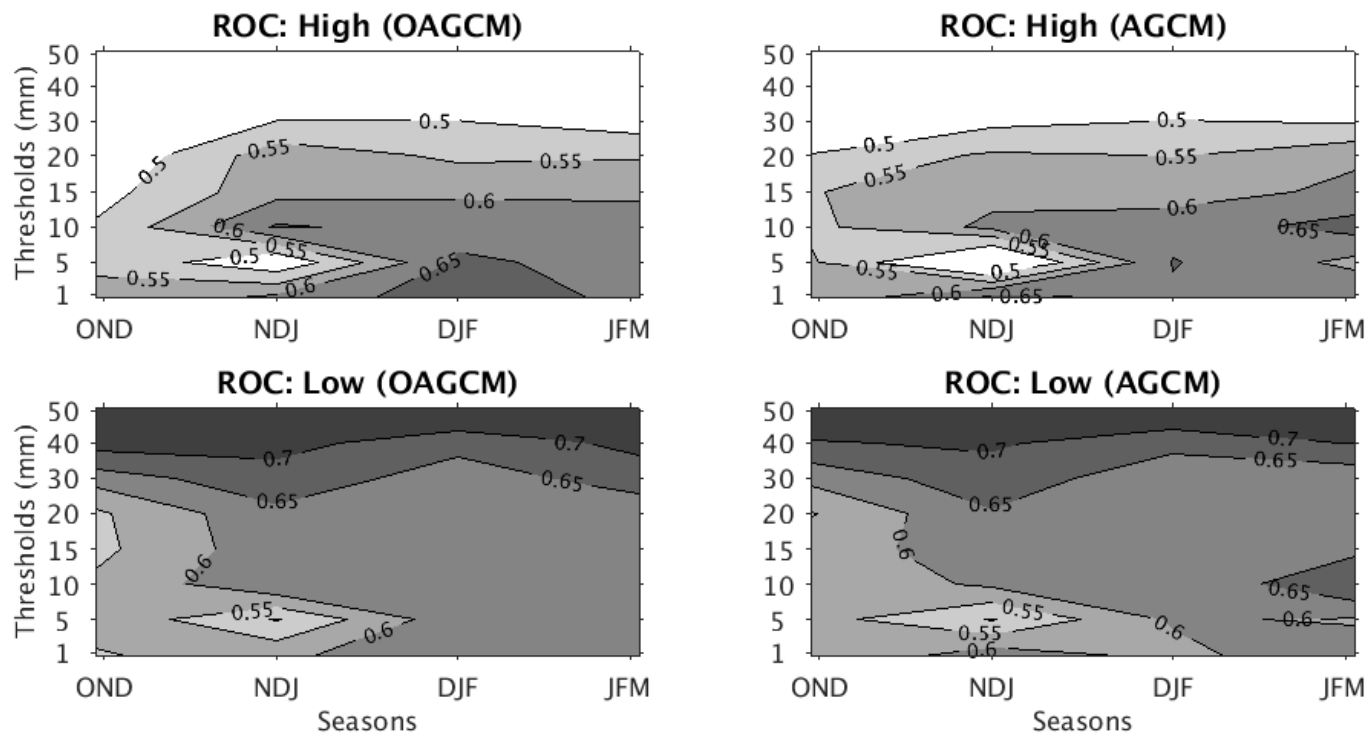

Figure 5. ROC scores for both the OAGCM (left panel) and the AGCM (right panel) at 1-month lead-time in predicting high- and low-number of rainfall days exceeding different threshold values over the 14 years retro-active forecasts from 1996 to 2009. 

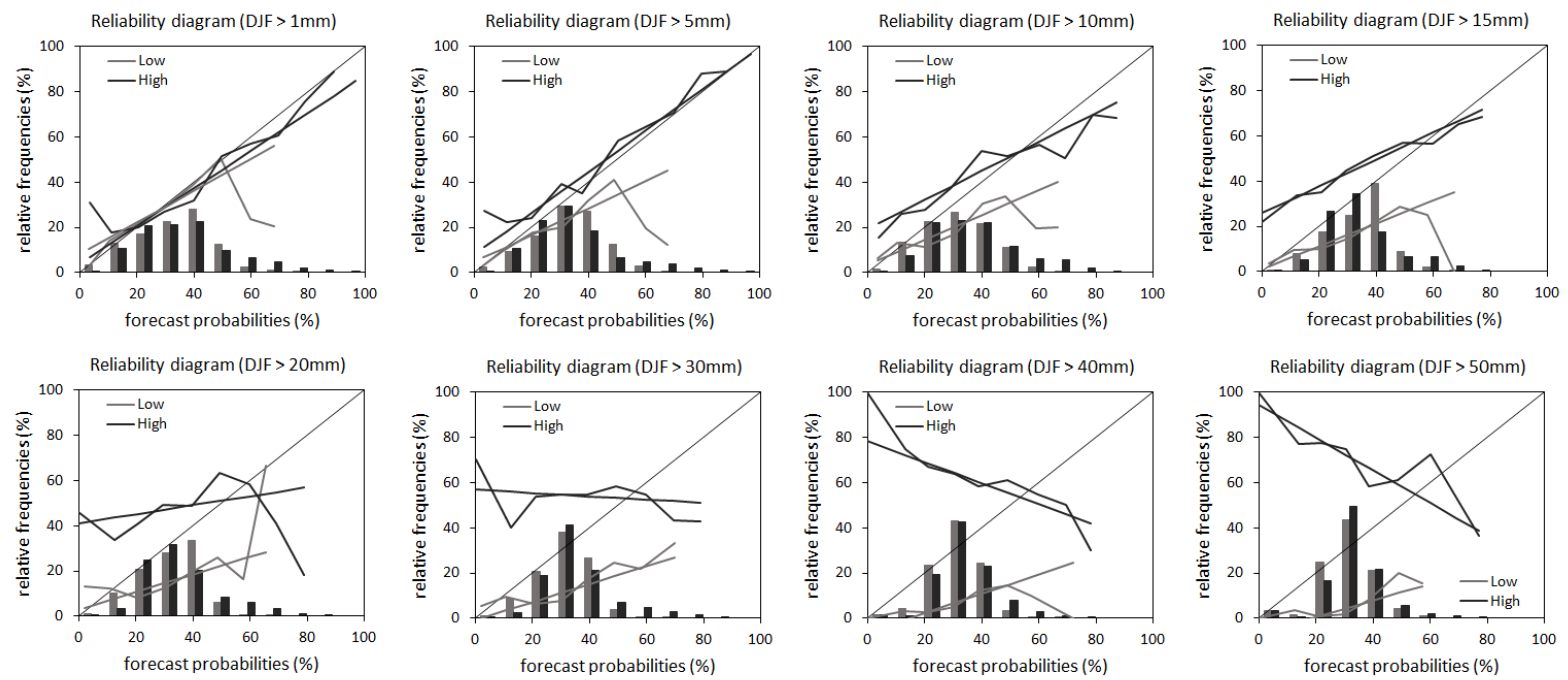

Figure 6. Reliability diagrams and frequency histograms for the OAGCM at 1-month lead-time in predicting highnumber and low-number of rainfall days exceeding pre-defined thresholds within DJF seasons over 14 years retroactive forecasts from 1996 to 2009.
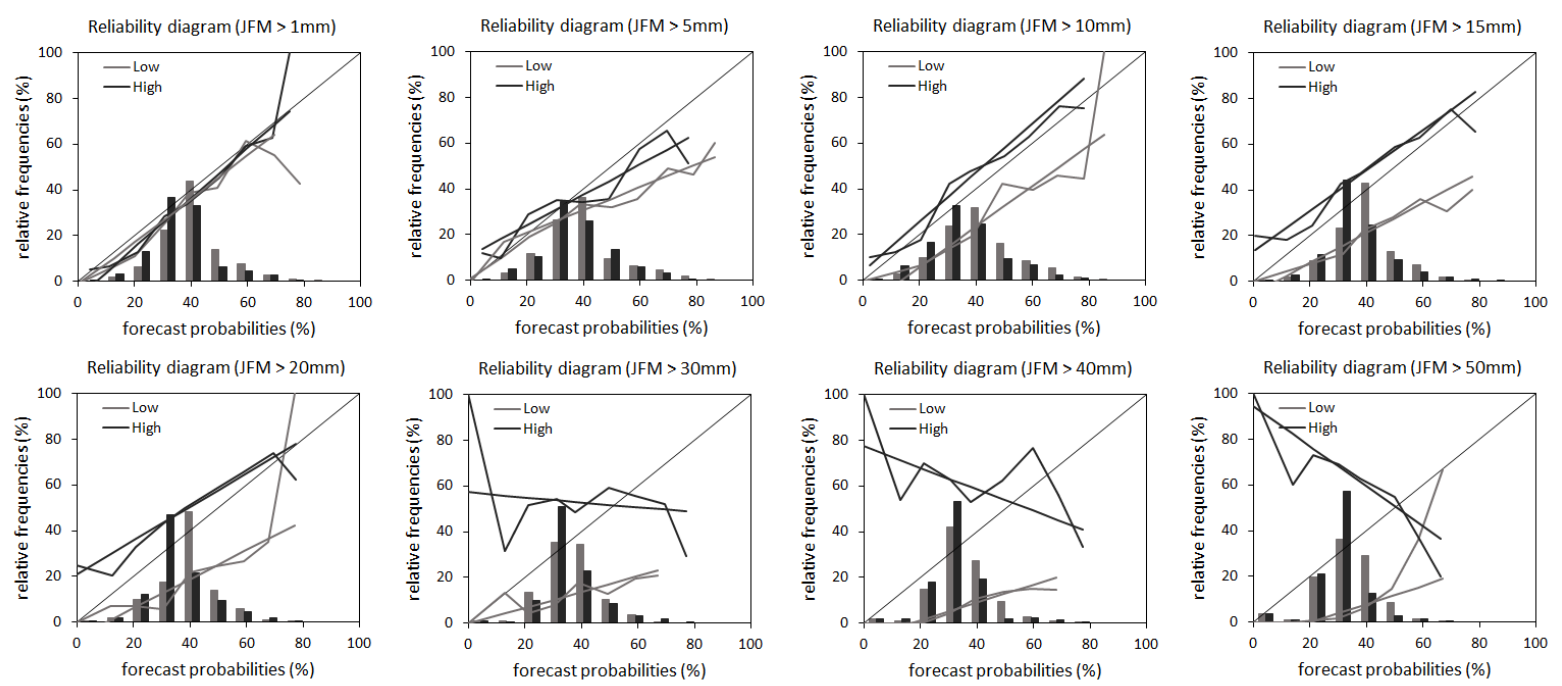

Figure 7. Reliability diagrams and frequency histograms for the AGCM at 1-month lead-time in predicting highnumber and low-number of rainfall days exceeding pre-defined thresholds within JFM seasons over 14 years retroactive forecasts from 1996 to 2009. 

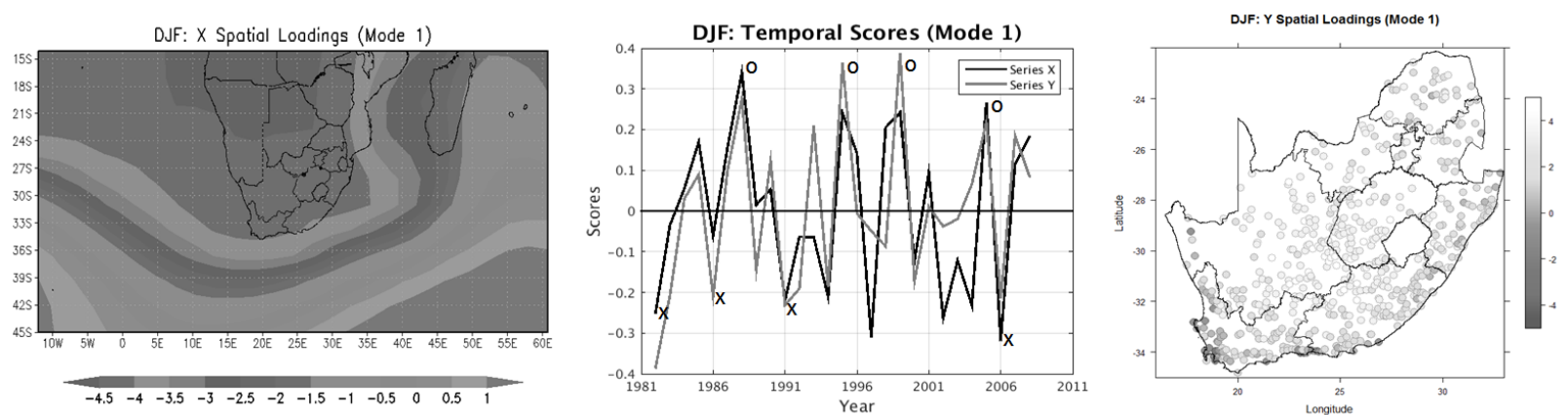

Figure 8. Mode $1 \mathrm{CCA}$ maps of the $850 \mathrm{hPa}$ geopotential heights of the AGCM at 1-month lead-time and the number of rainfall days exceeding $10 \mathrm{~mm}$ DJF seasons. The $\mathrm{X}$ and $\mathrm{Y}$ are the spatial loadings of the predictors and predictands, respectively.

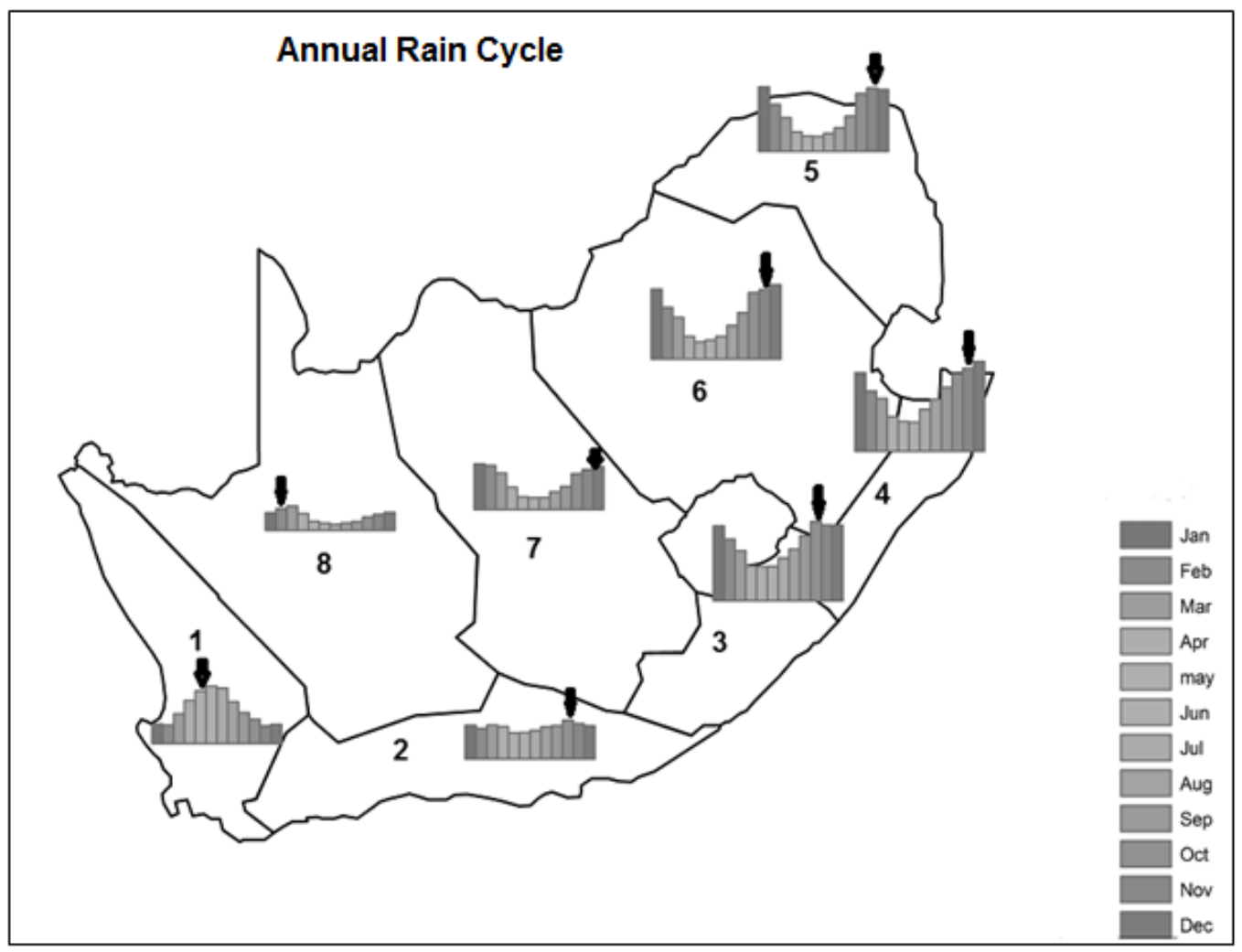

Figure 9. The eight homogeneous rainfall regions calculated using cluster analysis and their annual rainfall cycles from 1982 to 2009. 

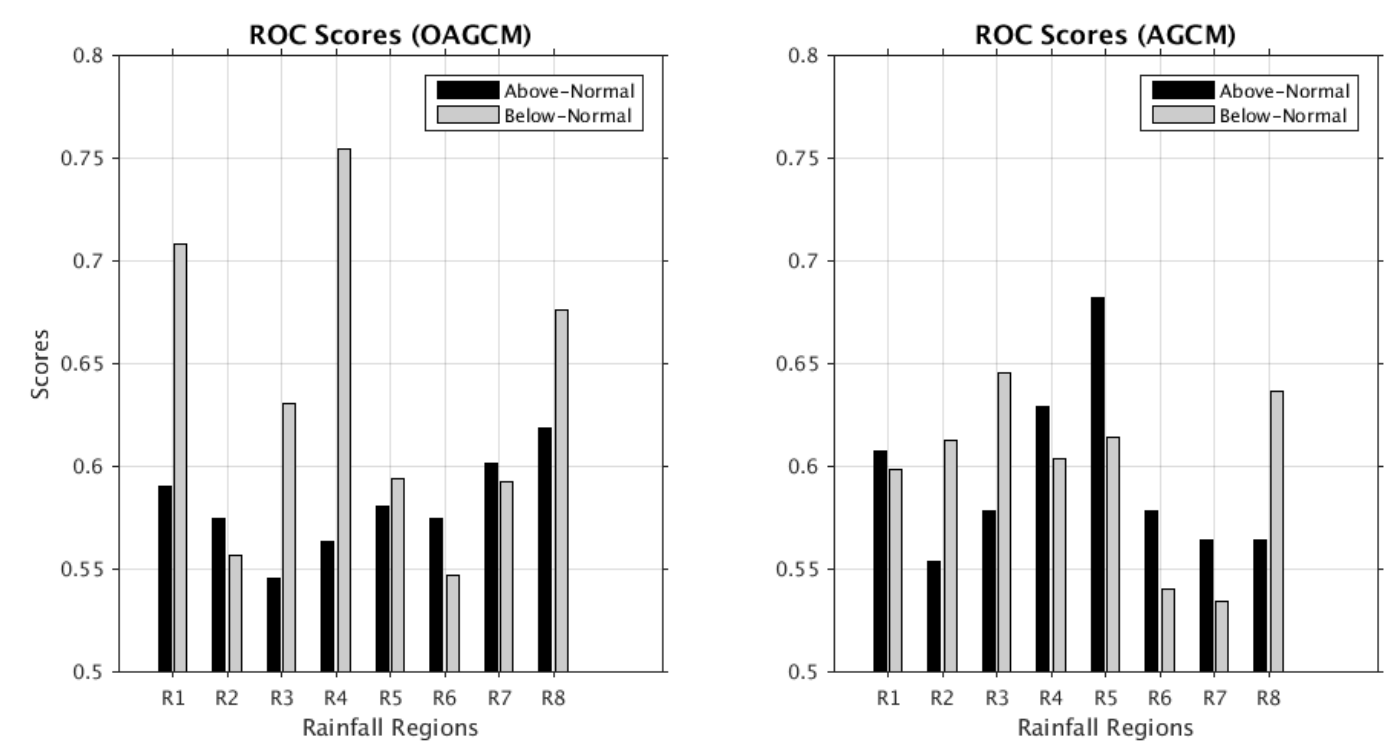

Figure 10. ROC scores of the forecasts downscaled from OAGCM (left) and AGCM (right) 1-month lead-time in predicting above-normal and below-normal rainfall totals of the onset months for the eight homogeneous rainfall regions of South Africa over the 14 year retro-active forecasts from 1996 to 2009.
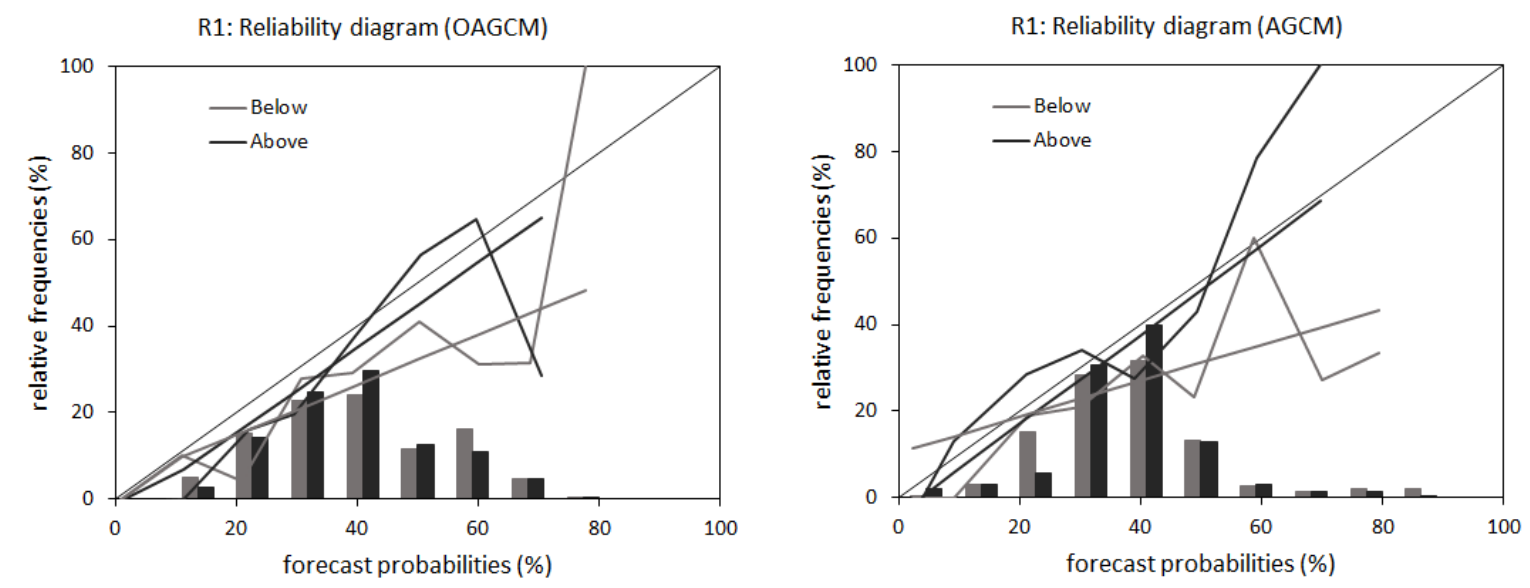

Figure 11. Reliability diagrams and frequency histograms for the downscaled forecasts produced by both the OAGCM (Coupled) and AGCM (Uncoupled) 1-month lead-time in predicting above-normal and below-normal rainfall totals of the onset month for Region 1 over 14 year retro-active forecasts from 1996 to 2009. 

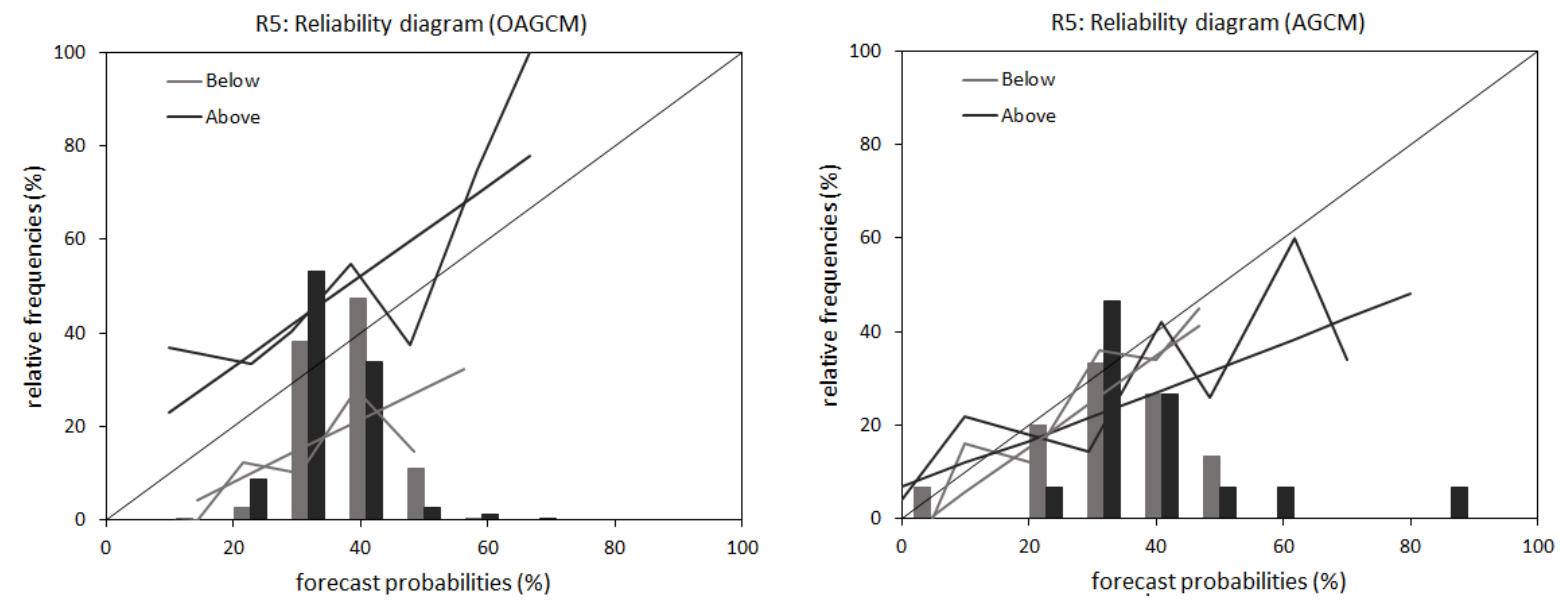

Figure 12. Reliability diagrams and frequency histograms for the downscaled forecasts produced by both the OAGCM (Coupled) and AGCM (Uncoupled) 1-month lead-time in predicting above-normal and below-normal rainfall totals of the onset month for Region 5 over 14 year retro-active forecasts from 1996 to 2009.
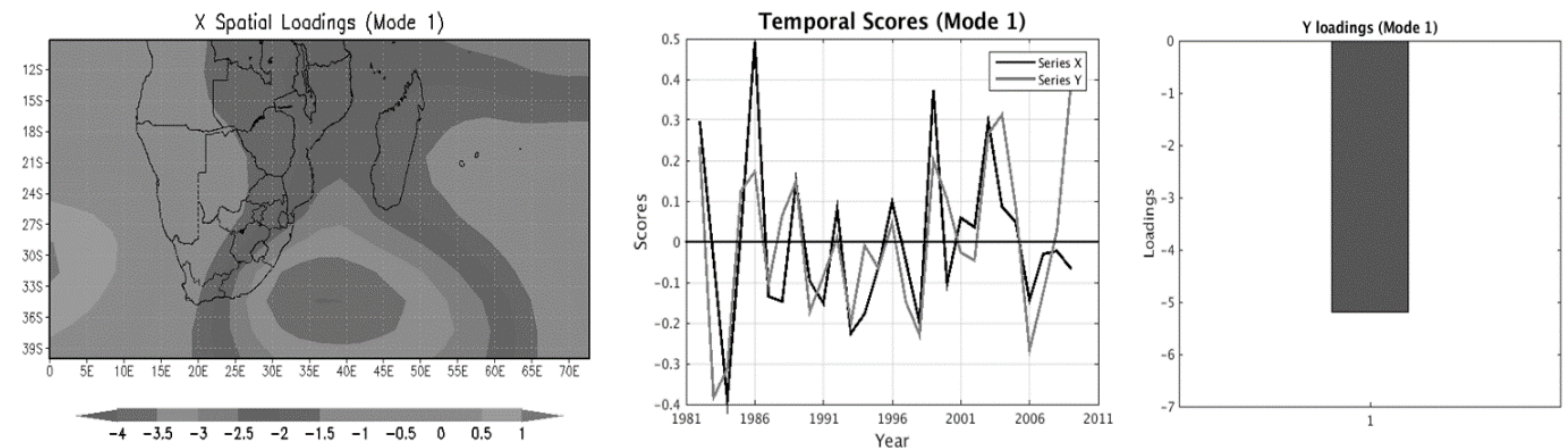

Figure 13. Mode $1 \mathrm{CCA}$ maps for the $850 \mathrm{hPa}$ geopotential heights of the OAGCM 1-month lead-time and rainfall totals for onset months for Region 1.
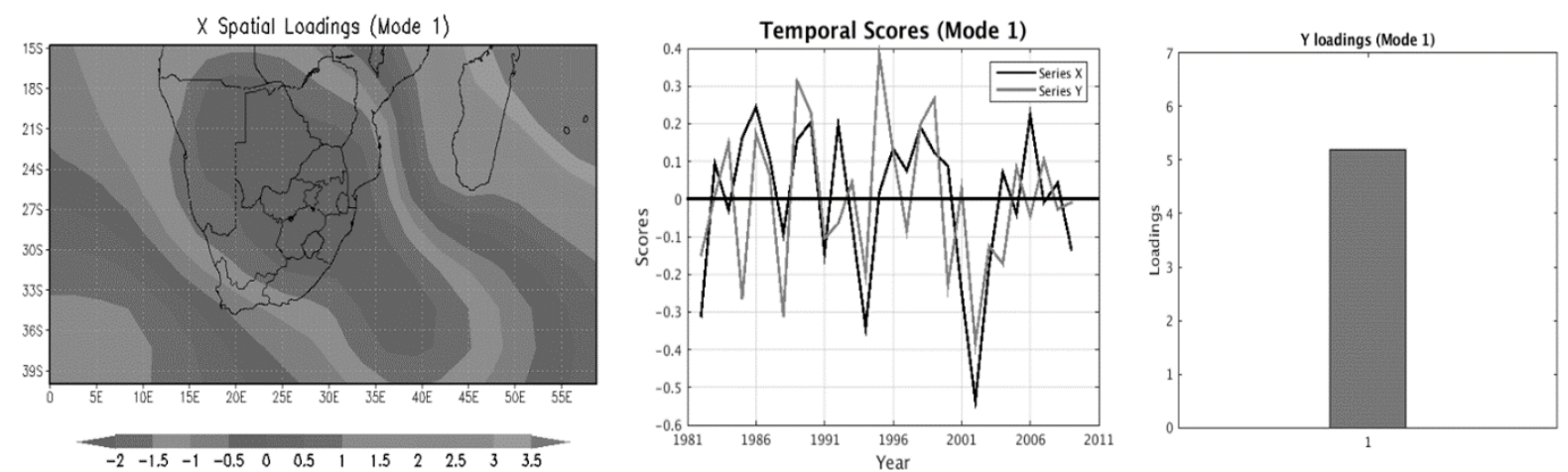

Figure 14. Mode 1 CCA maps for the 850hPa geopotential heights of the AGCM 1-month lead-time and rainfall totals for onset months for Region 5. 